\title{
A Prospective Randomized Pilot Study Evaluating the Scar Outcome after Gluteal Dermis Fat Graft with and without Kinesiotaping
}

\section{Annemarie Klingenstein ( $\sim$ Annemarie.Klingenstein@med.uni-muenchen.de )}

Ludwig-Maximilians-Universität München: Ludwig-Maximilians-Universitat Munchen https://orcid.org/0000-0001-8767-7660

\section{Aylin Garip-Kuebler}

Ludwig-Maximilians-Universität München: Ludwig-Maximilians-Universitat Munchen

\section{Daniel R Muth}

Ludwig-Maximilians-Universität München: Ludwig-Maximilians-Universitat Munchen

\section{Christoph Hintschich}

Ludwig-Maximilians-Universität München: Ludwig-Maximilians-Universitat Munchen

\section{Research Article}

Keywords: dermal fat graft, kinesiotaping, scar therapy, enucleation

Posted Date: October 18th, 2021

DOI: https://doi.org/10.21203/rs.3.rs-941204/v1

License: (c) (i) This work is licensed under a Creative Commons Attribution 4.0 International License. Read Full License

Version of Record: A version of this preprint was published at International Ophthalmology on April 2nd, 2022. See the published version at https://doi.org/10.1007/s10792-022-02304-7. 


\section{Abstract}

Purpose: To compare gluteal wound healing after dermis fat graft (DFG) implantation in patients with and without local application of kinesiotapes.

Methods: In this prospective, single-center analysis, 16 patients who underwent DFG implantation were randomized in two groups. Wound healing was compared 4-6 weeks after therapy and 3 months later (after application of 2 cycles of kinesiotaping for 2-3 weeks in the case and no specific therapy in the control group). Demographic data, patient content and wound healing were assessed. Scarring was graded (0-3) by evaluation of photodocumentation by 2 blinded, independent observers.

Results: Mean scar grading by both observers decreased from $2.31 \pm 0.48$ to $1.13 \pm 0.72$ in the case and from $2.38 \pm 0.52$ to $1.44 \pm 0.50$ in the control group with interobserver-agreement on scar grading being substantial to almost perfect in both groups. Scar length decreased significantly in both groups $(p=0.008)$. Scar prominence decreased in $2 / 3$ of cases in the case and $1 / 3$ in the control group. Scar coloring significantly improved in the case group alone $(p=0.031)$.

Conclusion: No functionally impairing or painful scar developed. No adverse effects occurred after kinesiotaping. Gluteal scars shortened significantly over time and were significantly paler in the case group. Kinesiotaping could prevent hypertrophic scarring or keloid formation.

Trial registration number: DRKS00023111; trial register: Deutsches Register Klinischer Studien (www.drks.de), retrospectively registered

\section{Introduction:}

Patients who require enucleation should be supplied with an orbital implant in order to obtain the best possible functional and cosmetic result. Dermis fat graft (DFG) implantation has been an established surgical procedure for decades [1] and excellent aesthetical and functional results regarding the socket are reported [2], [3]. An alternative procedure is enucleation and implantation of anorganic material with both porous and nonporous implants generally being well tolerated, and complication rates being low [4], [5]. Yet, long-term exposure of the implant is possible and rates may reach $24.7 \%, 23.5 \%$ and $76.5 \%$ for hydroxyapatite, bioceramic and Medpor ${ }^{\circledR}$, respectively [6], [7]. Our long-term clinical experience favors the results of autologous fat for implantation: We note a very low rate of conjunctival extrusion of the implant and of postenucleation syndrome as well as extremely rare rejection reactions due to the autologous implant material.

Yet, in comparison to alloplastic implants, the use of DFG results in possibly functionally and cosmetically restrictive scarring at the (gluteal or abdominal) donor site. In our clinic, we routinely perform DFG harvesting from the gluteal region at $5 \mathrm{~cm}$ above the middle of a connecting line between the anterior superior iliac spine and the ischial tuberosity [8]. 
In scarring, myofibroblasts produce collagen during healing. This process can be elevated and result in hypertrophic scarring or even keloid formation as a fibroproliferative disorder [9], resulting from a patient's skin type, healing tendencies or predispositions. In order to minimize post-surgical scar development, correct incision planning, skin closure, and postoperative care must be considered essential [10]. Different treatment algorithms for prophylaxis and therapy of hypertrophic scars or keloids are possible, but there is no established treatment strategy [11]. Early treatment possibilities include topical treatment with silicone (as sheets, gels, sprays or foams) [12], [13] as first-line therapy which decrease the scar size [14], [15], onion extract creams or oils as well as physical pressure or massaging with or without bandaging or taping to apply pressure [11].

Kinesiotaping is supposed to weaken subcutaneous adhesions and thus improve the appearance and softness of the scar. Physical therapists apply kinesiotapes tensionless over the complete length with its base in the center of the scar, oftentimes treating scars over joints. Up to now, only few studies have investigated the beneficial use of tapes to improve postoperative scarring [16], [17].

Hypertrophic scars that do not improve by 6 months should be managed intensively with intralesional steroid injections or alternate modalities [12]. Intralesional steroid injections reducing collagen production and thus increasing degradation within the fibrous portion of the scar are reportedly highly effective in the management of hypertrophic scars [18], [19]. Furthermore, laser therapy may flatten elevated scars and lighten pigmentation [20], [21] and cryotherapy and repeated surgery have been reported [11], [22].

In this prospective, randomized, mono-centric, single center analysis, we compared wound healing after gluteal DFG harvesting: Patients in the case group were treated by applying kinesiotape after initial evaluation of the scar 4-6 weeks after therapy whereas patients in the control group did not apply any treatment to the resulting scar. We hyposthesized that gluteal scarring can be improved functionally as well as cosmetically by kinesiotaping of the scar and hypertrophic scarring or keloid formation can thus be prevented.

\section{Methods:}

The ethics committee of Ludwig-Maximilians University, Munich / Germany approved the prospective, randomized, case-control evaluation of the gluteal wound healing process of patients having undergone enucleation and primary DFG implantation as performed in this trial (vote number 19-093, registration date 10/04/2019) at the oculoplastic department of the eye clinic of Ludwig-Maximilians University, Munich / Germany after evaluation and approval of the study protocol. The study is in accordance with the Declaration of Helsinki. Written informed consent was obtained from all patients prior to inclusion into the study. The study is registered in the Deutsches Register Klinischer Studien (www.drks.de; number DRKS00023111, registration date 05/10/2020).

Patient eligibility criteria included patients having undergone DFG harvesting over 18 years of age. Exclusion criteria were skin alterations as well as known allergic reactions to tape. 
The primary endpoint of the study was evaluation of the gluteal scar (length, color, prominence, indentation, visibility) 4-6 weeks post surgery as well as 3 months later. The secondary endpoint was patient content regarding functionality and cosmesis.

Sample size was calculated as per the expected number of surgeries over a period of two years.

Originally, inclusion of 50 patients was planned ( 5 sets of 10 numbers evenly randomized to the case and control groups by the Research Randomizer software (https://www.randomizer.org/). Numbers were sealed in sequentially numbered envelopes in order to conceal the sequence until interventions were assigned.

The patients in the case group locally applied kinesiotapes (hypo-allergenic spiral tapes from Atex Medical, CE certification as a medical product by Medical Device Directive 93/42/EEC seit 2007/47/EC, Standards ISO 10993-1, ISO 14971) starting after a first evaluation and documentation of the scar 4-6 weeks after surgery for 2 cycles of 2-3 weeks. These skin-colored tapes do not penetrate the epidermis, do not contain transdermal medication and function mechanically. Application of the tape is simple and approved for doctors as well as for physical therapists.

\section{Dermis fat graft harvesting}

Surgery was performed under general anaesthesia employing the technique described by Smith et al. [1]. In brief, the DFG with a standard size of $25 \mathrm{~mm}$ in diameter and thickness in adults was harvested from the gluteal region of the patients incising the epidermis superficially with a No. 15 scalpel, injection of saline intradermally at the donor site, then dissecting and separating the epidermis from the dermis layer by a No. 20 blade. Then, after deep transection of the fat layer, the dermis fat graft was explanted and the wound closed in two layers with three 2.0 absorbable subcutaneous sutures (Vicryl®, Ethicon, Johnson \& Johnson Medical GmbH, Norderstedt, Germany in both groups) after control of obvious bleeding using bipolar cautery. Closure of the skin was performed with 2.0 silk mattress sutures. Excision of "dog's ears" for better wound adaptation, if performed, was annotated for analysis.

At the donor site, steristrips were placed perpendicularly over the sutured wound followed by a sterile dressing for 2 days. Wound adaptation was then checked. Gluteal sutures were removed at 10-12 days after surgery.

\section{Application of kinesiotapes}

In the case group, the kinesiotapes were fitted over the clean scar without tension after having checked and documented proper wound healing thus far. They were then left in place for 2-3 weeks until they could be peeled off easily. Another cycle of kinesiotaping was applied if the scar was free of irritation (all cases). No further topical treatment was applied to the scar.

\section{Postoperative evaluation and assessment of the gluteal scar}


Postoperative follow-up visits were performed 4-6 weeks after surgery prior to first prescription of the prosthesis (V1), as well as another 3 months later (V2). At both points in time, overall patient content was elaborated (binary response noted, yes or no, respectively).

Wound healing was evaluated within the orbit and at the gluteal donor site of the DFG. Gluteal wound healing was documented by a still photographed close-up of the donor site at approximately $30 \mathrm{~cm}$ distance using a digital camera with a metric scale (calibration in $\mathrm{cm}$ and $\mathrm{mm}$ ) placed carefully next to the scar without covering any parts. The pictured gluteal section measured approximately $10 \times 10 \mathrm{~cm}$ and excluded the perianal region. Cosmetic evaluation of the scar was performed by two blinded observers separately using the same lighting and computer screen. The visibility of the scar was graded from grade 0 (scar not visible), 1 (minimally visible), 2 (moderately visble) and 3 (maximally visible) at both examinations. Additionally, total length at V1 and V2 as well as final color (erythema or normal skin color), prominence and indentation (presence or absence, respectively) of the scar were assessed.

Potential wound-healing confounders included patient gender (female or male), patient age at surgery, surgical resection of so-called "dog's ears" for better wound adaptation, patient body-mass-index (BMI), the surgeon $(1,2$, and 3$)$ and anticoagulation.

\section{Statistical analysis}

Demographic data (age group, sex, laterality as well as comparison of scar grading) were analysed by Fisher's exact test. The changes of the scar from baseline (4-6 weeks after surgery) to next follow-up (3 months later) were analysed by Wilcoxon signed rank test. Inter-observer reliability was compared by Kappa test. For all tests, $P<0.05$ was considered to be statistically significant.

\section{Results:}

Analysis was completed in all 16 patients, including 8 in both the case group ( 3 female), and 8 in the control group (4 female, no statistically significant difference, n.s.). Enucleation and primary implantation of an autologous DFG for this pilot-study was performed between 04/2019 and 10/2020 (study inclusion at V1 4- 6 weeks post surgery, 18 months recruitment period). In this interim analysis, we included 16 patients that were evenly randomized to the case and control group ( 1 set of 10 patients, 6 patients allocated from set 2) due to the lower number of operations performed during the global pandemic than originally assumed in the study protocol. Respective mean, median, and range of age at surgery were 52.7, 55.7, and 24.7-69.7 years for the case, and 58.0,60.8, and 24.0-85.0 years for the control group (n.s.). As the DFG is an autologous implant, a rejection reaction is rare and has not been reported within this patient collective.

V1 took place $44 \pm 16$ days post surgery in the case and $37 \pm 12$ days in the control group (n.s.). V2 was at $131 \pm 31$ days post surgery in the case and $125 \pm 16$ days in the control group (n.s.). Due to COVIDrelated quarantine, one patient of the case and one patient of the control group had to postpone their originally scheduled V2 and V1, respectively. Patient height was $173,8 \pm 9.0 \mathrm{~cm}$ in the case and $172.3 \pm$ 
$13.0 \mathrm{~cm}$ in the control group (range $165-184 \mathrm{~cm}$ and $154-189 \mathrm{~cm}$, respectively, n.s.). Patient weight was $83.3 \pm 11.7 \mathrm{~kg}$ in the case and $83.8 \pm 27.4 \mathrm{~kg}$ in the control group (range $70-97 \mathrm{~kg}$ and $53-130 \mathrm{~kg}$, respectively, n.s.). This resulted in a body mass index (BMI) of $27.1 \pm 3.3 \mathrm{~kg} / \mathrm{m}^{2}$ in the case and $28.3 \pm$ $8.9 \mathrm{~kg} / \mathrm{m}^{2}$ in the control group (range $22.0-32.0 \mathrm{~kg} / \mathrm{m}^{2}$ and $20.0-48.0 \mathrm{~kg} / \mathrm{m}^{2}$, respectively, n.s.).

No patient had previous history of hypertrophic scar formation. One patient of the control group was operated under systemic anticoagulation (100mg aspirin daily). Group comparison of the surgeon performing the operation (1-3) and performing wound closure with so-called "dog's ears" (in 4 patients per group) to improve wound adaptation was not significant, respectively.

Enucleation with DFG harvesting was performed due to painful amaurosis in 8 , uveal melanoma in 6 (recurrence in 3 and secondary glaucoma in 3), buphthalmos in 1 and posttraumatic perforation of the globe in 1 case(s). No patient presented with postoperative complications as infection, bleeding or reported any functional motility restriction resulting from the scar at V1 or V2 (yes or no answer modality). In the case group, no skin alterations resulting from kinesiotaping were observed.

Mean scar grading at V1 and V2 by observers 1 and 2, total scar length in mm, noted color, scar prominence or indentation (yes or no, respectively) and visibility grading at V1 and V2 are shown in Table 1.

Table 1

Scar parameters and grading. \pm SD, $95 \%$ confidence interval in parenthesis; ${ }^{*} p<0.05$ Wilcoxon signed rank test.

\begin{tabular}{|lllll|}
\hline Scar parameters & $\begin{array}{l}\text { Case group } \\
\text { V1 }\end{array}$ & V2 & $\begin{array}{l}\text { Control group } \\
\text { V1 }\end{array}$ & V2 \\
\hline $\begin{array}{l}\text { Mean scar } \\
\text { length [mm] }\end{array}$ & 4.16 & $3.79^{*}$ & 4.10 & $3.88^{*}$ \\
\hline Prominence & $37.5 \%$ & $12.5 \%$ & $37.5 \%$ & $25.0 \%$ \\
\hline Colour & $100 \%$ & $37.5 \%^{*}$ & $87.5 \%$ & $62.5 \%$ \\
$\begin{array}{l}\text { Mean grading } \\
0-3\end{array}$ & $2.38 \pm 0.52$ & $1.13 \pm 0.64$ & $2.38 \pm 0.52$ & $1.38 \pm 0.74(0.75-$ \\
Observer 1 & $(1.94-2.81)$ & $(0.59-1.66)$ & $(1.94-2.81)$ & $2.00)$ \\
Observer 2 & $(1.85 \pm 0.46$ & $1.13 \pm 0.83$ & $2.38 \pm 0.52$ & $1.50 \pm 0.76$ \\
Mean Obs. 1+2 & $2.31 \pm 0.48$ & $(0.43-1.82)$ & $(1.94-2.81)$ & $(0.87-2.13)$ \\
\hline
\end{tabular}

Mean scar grading by the 2 observers decreased from $2.31 \pm 0.48(\mathrm{~V} 1)$ to $1.13 \pm 0.72(\mathrm{~V} 2)$ in the case and from $2.38 \pm 0.52(\mathrm{~V} 1)$ to $1.44 \pm 0.50$ (V2) in the control group. Grading decreased more in the case group, yet this was not significant ( 0.504 ; Fisher's exact test). Scar grading improved from V1 to V2 in 8 / 8 cases (observer 1) and 7 / 8 cases (observer 2) in the case and in 6 / 8 cases graded (both observers) in 
the control group. This was statistically significant (observer $1 p=0.008$ and $2 p=0.016$ case group and observer 1 and $2 p=0.031$ control group, respectively; Wilcoxon signed rank test). Interobserver agreement evaluated by Kappa test was 0.714 at V1 for the case and 1.000 for the control group and 0.610 and 0.784 at $\mathrm{V} 2$ for the case and control group, respectively (with strength of agreement of Cohens Kappa being substantial at 0.61-0.80 and almost perfect at 0.81-1.00). [23] Scar length decreased significantly in both groups over time from V1 to V2 ( $p=0.008$ (mean change $0.38 \mathrm{~mm}, 9.4 \%$ ) case group, $p=0.008$ (mean change $0.23 \mathrm{~mm}, 6.0 \%$ ) control group, Wilcoxon signed rank test, respectively). Scar prominence decreased in 2 out of 3 cases in the case and in 1 out of 3 cases in the control group from V1 to V2. Scar color decreased significantly from V1 to V2 in the case group $(p=0.031)$, but not in the control group ( $p=0.500$; Wilcoxon signed rank test, respectively). Figures $1 \mathbf{a}+\mathbf{b}$ and $\mathbf{2} \mathbf{a}+\mathbf{b}$ give an example of scar devolution with and without kinesiotaping.

No patient of either the case or control group reported dissatisfaction with the functional or aesthetic result of the scar at V1 or V2.

\section{Discussion:}

Although DFG harvesting is a routine surgical intervention, we could not find any data on prospective evaluation of the resulting scar at the donor site in common medical databases. In our opinion, such data is of great interest, as patients' expectations are continuously growing. Thus, focus on function and cosmesis is mandatory.

The most important results of this study include that, apparently, kinesiotaping of the gluteal scar after DFG harvesting leads to significant decrease of scar color at 3 months after the beginning of taping compared to the control group. Additionally, the scars significantly shortened after this time interval in both patient groups. Scar prominence improved in $2 / 3$ of cases in the case and $1 / 3$ of patients in the control group. Scar grading improved gradually in most patients of both groups (with inter-observer variability being substantial to almost perfect).

Hypertrophic scars must still be considered a challenge in wound rehabilitation with different treatment options available. Treatment should be based on scar location, quality, size as well as patient specific factors as preferences, and expectations [24]. Besides cosmetical problems, functional impairment such as contractures and subjective symptoms as pruritus may significantly affect the patients' quality of life [9].

Following the recent understanding, wound healing includes the inflammatory ( $2-3$ days), the proliferative (3-6 weeks) and the remodeling phase ( $>1$ year) which partly overlap [9], [12]. Scar management is intimately connected to these stages [12]. The remodeling phase is at its peak within the first three months after surgery in which incisions destined to develop hypertrophic response will begin to reveal themselves [12]. Once hypertrophic scarring begins, early intervention is crucial. 
We found the recommendation to continue dressing of the incision with skin tape during this phase in a review on surgical scar prevention and management [12] and therefore started with application of kinesiotapes as prophylaxis of hypertrophic scar and keloid formation after V1 (after 4-6 weeks post surgery). During this early remodeling phase, extracellular matrix as an immature healing component is remodeled and type III collagen ultimately matures to type I collagen [9].

Pressure therapy may prevent scar elevation [25] and is advised soon after clinical wound healing. One advantage of kinesiotaping over pressure therapy may be superior patient compliance, as pressure therapy exceeding $24 \mathrm{mmHg}$ for more than 30 minutes daily as recommended [12] is perceived as physically uncomfortable.

Despite pressure treatment ranking among the most common treatments, pressure garments may not be able to exert adequate pressure due to the complexity of the human body [26]. This is also the case for the scar after gluteal DFG harvesting: The location is under movement and mechanical contact of different layers of clothing. We thus consider it an advantage of kinesiotaping of the gluteal location that the tape effectively mobilizes the hypertrophic skin by consistently moving it against subcutaneous collagenous tissue.

Kinesiotaping is a non-invasive prophylactic as well as therapeutic procedure to improve scar formation with minor adverse effects. In this study, no hypersensitivity reactions of complications due to taping were reported. Application of kinesiotape is easier for the patient than application of silicone (which can be considered among first-line treatments of hypertrophic scars). Kinesiotaping is fitted over the scar without tension and can be left in place for 2-3 weeks while silicone sheets must be worn over the scar area for 12-24 hours daily for 2-3 months of applied multiple times per day as gel [11].

Inference from this study is limited in different ways: First, enucleation is not a frequent intervention with globe-preserving therapy being possible in many cases and surgery without harvesting of a DFG is possible, such that respective numbers of cases and controls were rather limited despite the 18-month recruitment-period of this prospective, randomized study at a large university center. Furthermore, despite photodocumentation excluding the perianal region, two patients refused participation in the study for reasons of modesty and two patients were incapacitated adults.

Second, patient compliance regarding the application of the tape was assumed as explained within the form of written informed consent but could not be controlled.

Third, scarring is not terminated at 17-19 weeks following surgery, but the scar will mature as the remodeling phase continues. Yet, good results could already be achieved within this limited timeframe and in hope of best patient compliance and associated quality of life, we tried to integrate the scar therapy within routine follow-up visits at our institution. Kinesiotaping could prevent hypertrophic scarring or keloids at an early stage. Longterm follow-up and comparison is warranted. 
No functionally impairing or painful scarring ocurred in the case or control group proving that in addition to the advantages of an autologous implant (low rate of conjunctival extrusion and postenucleation syndrom, good functional and aesthetic longterm outcome, extremely rare rejection rate), DFG harvesting is not a functional disadvantage in patient treatment over enucleation and supply with an alloplastic implant. Our preliminary results show good reduction of color, scar length and prominence already at 4-5 months post surgery. Kinesiotaping is a cost-efficient tool that is easily applicable and may aid in preventing hypertrophic scarring or keloid formation after gluteal DFG harvesting. This should be validated in further studies with larger patient collectives and longer follow-up.

\section{Declarations}

Financial and none-financial interests: The authors have no relevant financial or non-financial interests to disclose.

Conflicts of interest: No conflicting relationship exists for any author.

Availability of data and material: The blinded datasets used and analyzed during the current study are available from the corresponding author upon reasonable request.

Authors' contributions: $\mathrm{AK}, \mathrm{AGK}, \mathrm{DM}$ and $\mathrm{CH}$ all made substantial contributions to conception and design, acquisition of data, or analysis and interpretation of data; took part in drafting the article or revising it critically for important intellectual content; agreed to submit to the current journal; gave final approval of the version to be published; and agree to be accountable for all aspects of the work.

Ethics approval and consent to participate: The study was approved by the ethics committee of LudwigMaximilians-University, Munich, Germany (vote number 19-093). All patients gave written informed consent prior to inclusion into the study. All patient data is non-identifiable and complied with relevant data protection and privacy regulation.

Consent for publication: Not applicable, no identifiable images included.

\section{References}

1. Smith B, Bosniak SL, Lisman RD (1982) An autogenous kinetic dermis-fat orbital implant: an updated technique. Ophthalmology 89(9):1067-1071. https://doi.org/10.1016/s01616420(82)34690-4

2. Nentwich MM, Schebitz-Walter K, Hirneiss C, Hintschich C (2014) Dermis fat grafts as primary and secondary orbital implants. Orbit 33(1):33-38. https://doi.org/ 10.3109/01676830.2013.844172

3. Jovanovic N, Carniciu AL, Russell WW, Jarocki A, Kahana A (2020) Reconstruction of the orbit and anophthalmic socket using the dermis fat graft: A Major Review. Ophthalmic Plast Reconstr Surg. 26(6), 529-539. https://doi.org/10.1097/IOP.0000000000001610 
4. Wladis EJ, Aakalu VK, Sobel RK, Yen MT, Bilyk JR, Mawn LA (2018) Orbital implants in enucleation surgery: A report by the American Academy of Ophthalmology. Opthalmology. 125(2), 311-317. https://doi.org/10.106/j.ophtha.2017.08.006

5. Trichopoulos N, Augsburger JJ (2005) Enucleation with unwrapped porous and nonporous orbital implants: a 15-year experience. Ophthalmic Plast Reconstr Surg. 21(5), 331-336. https://doi.org/10.1097/01.iop.0000175034.88019.a5

6. Lin CW, Liao SL (2017) Long-term complications of different porous orbital implants: a 21-year review. Br J Ophthalmol 101(5):681-685. https://doi.org/ 10.1136/bjophthalmol-2016-308932

7. Guthoff R, Vick HP \& Schaudig U (1995) Prevention of postenucleation syndrome: the hydroxylapatite silicone implant. Preliminary experimental studies and initial clinical experiences. Ophthalmologe 92(2):198-205.

8. Hintschich C (2017) Dermis-Fett-Transplantation. Ophthalmologe 114(8):755-758. https://doi.org/10.1007/s00347-017-0528-5

9. Lee HJ, Jang Y (2018) Recent Understandings of Biology, Prophylaxis and Treatment Strategies for Hypertrophic Scars and Keloids. Int J Mol Sci 19(3):711. https://doi.org/10.3390/ijms19030711

10. Lee Peng G, Kerolus JL (2019) Management of surgical scars. Facial Plast Surg Clin North Am. 27(4), 513-517. https://doi.org/10.1016/j.fsc.2019.07.013

11. Cafasso J, Hannan C (2017) https://www.healthline.com/health/hypertrophic-scartreatment\#treatment. Accessed 27 December 2020

12. Son D, Harijan A (2014) Overview of surgical scar prevention and management. J Korean Med Sci 29(6):751-757. https://doi.org/10.3346/jkms.2014.29.6.751

13. Sawada Y, Sone K (1990) Treatment of scars and keloids with a cream containing silicone oil. Br J Plast Surg 43(6):683-688. https://www.doi.org/ 10.1016/0305-4179(90)90008-k

14. Chang CC, Kuo YF, Chiu HC, Lee JL, Wong TW, Jee SH (1995) Hydration, not silicone, modulates the effects of keratinocytes on fibroblasts. J Surg Res 59(6):705-711. https://www.doi.org/10.1006/jsre.1995.1227

15. Borgognoni L (2002) Biological effects of silicone gel sheeting. Wound Repair Regen 10(2):118-21. https://www.doi.org/10.1046/j.1524-475x.2002.00205.x

16. Atkinson JM, McKenna KT, Barnett AG, McGrath DJ, Rudd M (2005) A randomized, controlled trial to determine the efficacy of paper tape in preventing hypertrophic scar formation in surgical incisions that traverse Langer's skin tension lines. Plast Reconstr Surg. 116(6), 1648-1656. https://www.doi.org/10.1097/01.prs.0000187147.73963.a5

17. Lin Y, Ting P, Hsu K (2020) Comparison of silicone sheets and paper tape for the management of postoperative scars: A randomized comparative study. Adv Skin Wound Care. 33(6), 1-6. https://www.doi.org/10.1097/01.ASW.0000661932.67974.76d

18. Tang YW (1992) Intra- and postoperative steroid injections for keloids and hypertrophic scars. $\mathrm{Br} \mathrm{J}$ Plast Surg 45(5):371-373. https://www.doi.org/10.1016/0007-1226(92)90007-k 
19. Rosen DJ, Patel MK, Freeman K, Weiss PR (2007) A primary protocol for the management of ear keloids: results of excision combined with intraoperative and postoperative steroid injections. Plast Reconstr Surg 120(5):1395-1400. https://www.doi.org/10.1097/01.prs.0000279373.25099.2a

20. Bouzari N, Davis SC, Nouri K (2007) Laser treatment of keloids and hypertrophic scars. Int J Dermatol 46(1):80-88. https://www.doi.org/10.1111/j.1365-4632.2007.03104.x

21. Parrett BM, Donelan MB (2010) Pulsed dye laser in burn scars: current concepts and future directions. Burns 36(4):443-449. https://www.doi.org/ 10.1016/j.burns.2009.08.015

22. nhs (2017) https://www.nhs.uk/conditions/scars/treatment/. Accessed 28 December 2020

23. Landis JR, Koch GG (1977) The Measurement of Observer Agreement for Categorical Data. Biometrics 33(1):159-174

24. Shin J, Cho JT, Park SI, Jung SN (2019) Combination therapy using non-ablative fractional laser and intralesional triamcinolone injection for hypertrophic scars and keloids treatment. Int Wound $\mathrm{J}$ 16(6):1450-1456. https://www.doi.org/ 10.1111/iwj.13213.

25. Niessen FB, Spauwen PH, Schalkwijk J, Kon M (1999) On the nature of hypertrophic scars and keloids: a review. Plast Reconstr Surg 104(5):1435-1458. https://www.doi.org/10.1097/00006534199910000-00031

26. Chow L, Yick KL, Kwan MY, Yuen CF, Ng SP, Yu A, et al (2020) Customized Fabrication Approach for Hypertrophic Scar Treatment: 3D Printed Fabric Silicone Composite. Int J Bioprint 6(2):262. https://www.doi.org/10.18063/ijb.v6i2.262

\section{Figures}

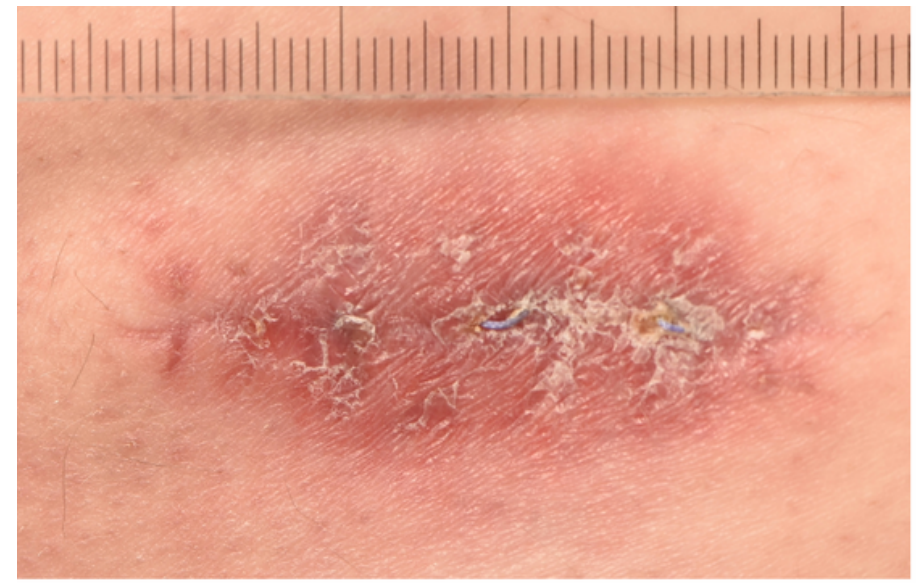

A

\section{Figure 1}

Gluetal scar without specific therapy. Scar graded as 3 / 3 (observer 1 / 2) at a) V1 (33 days after surgery; $3.5 \mathrm{~cm}$ ) and as $2 / 2$ at b) V2 (119 days after surgery; $3.4 \mathrm{~cm}$ ) without kinesiotaping. 


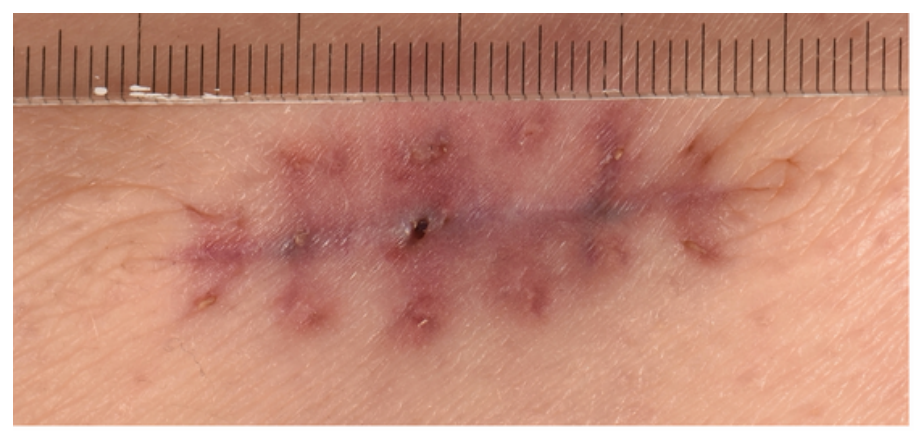

A

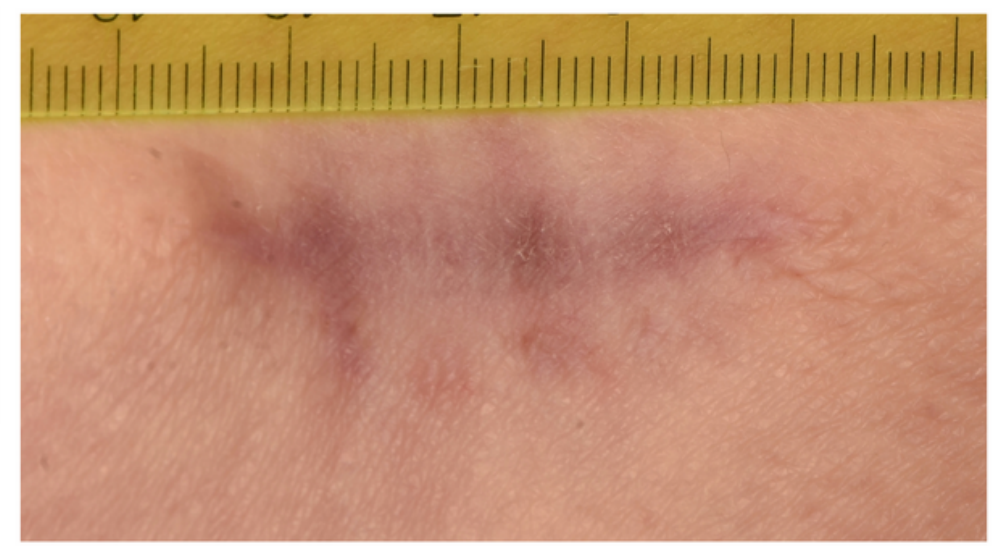

B

\section{Figure 2}

Gluteal scar treated with kinesiotapes. Scar graded as 3 / 3 at a) V1 (also 33 days after surgery; length $3.9 \mathrm{~cm}$ ) and graded as $1 / 2$ at b) V2 (124 days after surgery; length $3.6 \mathrm{~mm}$ ) after application of 2 cycles of kinesiotaping demonstrating less scar coloring at V2.

\section{Supplementary Files}

This is a list of supplementary files associated with this preprint. Click to download.

- CONSORTChecklistDFG.pdf

- ConsortflowchartDFG.pdf 